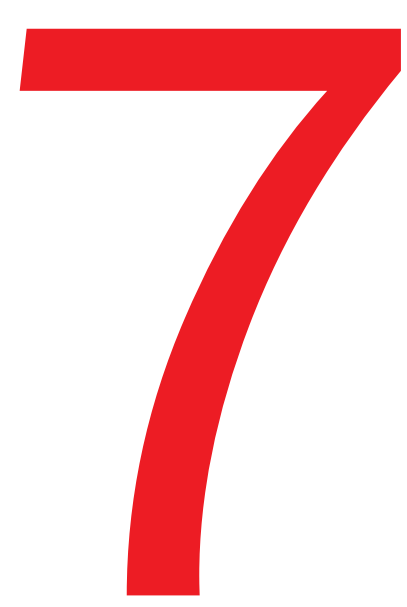

\title{
Résumé
}

L'histoire de la discipline infirmière démontre des efforts progressifs en vue de développer du savoir infirmier. Cet article cible la science infirmière en postulant qu'il s'agit d'une partie de la discipline infirmière et d'une partie du savoir de la discipline. Dans ce contexte, une attention particulière est portée aux théories en tant que produit de la science et elles seront situées dans l'avancement de la science infirmière. L'examen de la science infirmière et de son évolution s'inspire de deux visions de la structure du savoir de la discipline infirmière. L'étude de l'évolution de la discipline sur le plan scientifique est abordée en trois périodes. Cet examen est complété par les propositions de deux métathéoriciens se préoccupant des théories dans l'avancement du savoir. En conclusion, au-delà des différences de propositions au sujet du savoir scientifique et de son évolution, on voit qu'une structure du savoir joue un rôle durable.

Mots-clés développement, discipline, science, structure, théorie

\section{Évolution de la dimension scientifique de la discipline infirmière : quelques repères}

\author{
FARZANEH FARSHBAF KAMEL \\ \& CLÉMENCE DALLAIRE
}

\section{Introduction}

La discipline infirmière, branche du savoir humain, s'intéresse à des phénomènes qui découlent des intérêts centraux du domaine.[1,2,3] Nous postulons qu'une discipline se compose de plusieurs dimensions et qu'il est possible de cibler l'étude de l'évolution d'une seule d'entre elles. Pour les besoins de cet article, nous avons ciblé la dimension scientifique. Même s'il n'existe pas de définition unique de ce qu'est la science, nous constatons que les efforts des théoriciens et des métathéoriciens ont été influencés par différentes visions de la science. En dépit des différences de conceptions de la science, on note que la majorité de ces conceptions accordent une place importante aux théories. Puisque l'examen des théories et de l'avancement de la science infirmière représentent une vaste question et constituent une entreprise qui dépasse largement les visées du présent article, nous allons restreindre le sujet à la contribution des théories dans le développement de la science infirmière. En somme, cet article se concentre sur certaines questions portant sur la discipline infirmière et propose des réponses provisoires au sujet de l'avancement de l'aspect scientifique de cette dernière.

\section{Structures du savoir infirmier}

Les soins infirmiers sont une discipline professionnelle et une discipline organise son savoir d'une certaine façon. En corollaire de ces prémisses, la science infirmière, en tant que composante de la discipline infirmière, s'inspire de visions de la science et de ses méthodes. Dans cette perspective, le savoir scientifique a pris et prend encore la forme de 
théories, une théorie étant considérée comme un indicateur important de la science. Avant de poursuivre l'examen de la science infirmière et des théories, notons que les structures proposées pour organiser le savoir infirmier sont nombreuses et nous en retenons deux qui sont complémentaires. Elles se présentent davantage en complémentarité qu'en contraste puisqu'elles mettent l'accent sur des éléments différents. Ces deux visions de la structure du savoir infirmier seront abordées succinctement afin de bien situer la science infirmière dans la discipline. La première structure a été proposée très tôt dans l'évolution récente de la discipline infirmière et elle estaussitrès générale. Elle offre l'avantage d'aider à saisir les éléments qui vont permettre d'étudier l'évolution de la discipline infirmière en ciblant sa seule dimension scientifique. Selon Donaldson et Crowley[1], les disciplines ont une structure composée à la fois de substance et de syntaxe. La substance, soit les conceptualisations de phénomènes qui représentent ou sont en adéquation avec la perspective de la discipline, correspond au savoir. La syntaxe correspond aux méthodologies utilisées pour examiner les phénomènes pertinents afin de proposer du savoir au sujet de ces phénomènes et correspond aux critères utilisés pour accepter ce savoir (Figure 1). Au fil des travaux de recherche [syntaxe], on voit apparaitre des théories dans une discipline [substance], théories qui représentent des explications, des descriptions ou des prédictions au sujet des phénomènes étudiés. C'est grâce à ces deux composantes, la substance et la syntaxe, que l'on peut particulièrement cerner le savoir infirmier scientifique qui représente une partie du savoir de la discipline. Comme le savoir est constitué de différents éléments, et dans le cas du savoir scientifique, de substance et de syntaxe, il est nécessaire de recourir à une façon de l'organiser. Pour cette raison, la proposition de Donaldson et Crowley[1] met de l'avant l'examen de la dimension scientifique de la discipline infirmière en situant les théories dans une structure du savoir et en suggérant un processus général de leur développement.

La deuxième structure que nous retenons est celle proposée par Schlotfeldt[4] qui propose d'organiser le savoir de la discipline infirmière au moyen d'une grande sphère ayant une membrane perméable et extensible. Schlotfeldt4] organise en sphère les types de connaissances de la discipline en soulignant la " science infirmière " dans cette représentation (Figure 3). Selon Schlotfeldt[4], la discipline infirmière contient les cinq types de connaissances suivantes: a] la science infirmière; b] l'histoire des soins infirmiers; c] la philosophie infirmière; d] les interventions infirmières; et e] les facteurs influençant la santé humaine. Les flèches indiquent que ces composantes se complètent et ne sont pas isolées les unes des autres. Dans cette structure [Figure 3], le plus grand segment de la sphère représente le savoir scientifique des soins. La science infirmière comprend des principes et des lois découvertes par la recherche scientifique qui sont pertinentes et utiles pour la pratique.[4] En plus, elle contient les théories scientifiques existantes formulées par les infirmiers théoriciens qui guident les recherches scientifiques sur les soins et représentent les explications prometteuses au sujet de phénomènes particuliers aux soins.

Selon ces deux visions de la structure de la discipline, celle de Donaldson et Crowley[1] et celle de Schlotfeldt[4], on voit bien que la discipline infirmière peut se concevoir en différentes composantes et qu'ainsi, on peut y distinguer, au sein de la substance disciplinaire, une partie du savoir que nous appelons la science infirmière. Ce qui précède nous permet d'avancer que le savoir infirmier inclut la science infirmière en tant que partie importante du savoir de la discipline, mais que le savoir de la discipline est plus large que le seul savoir scientifique.

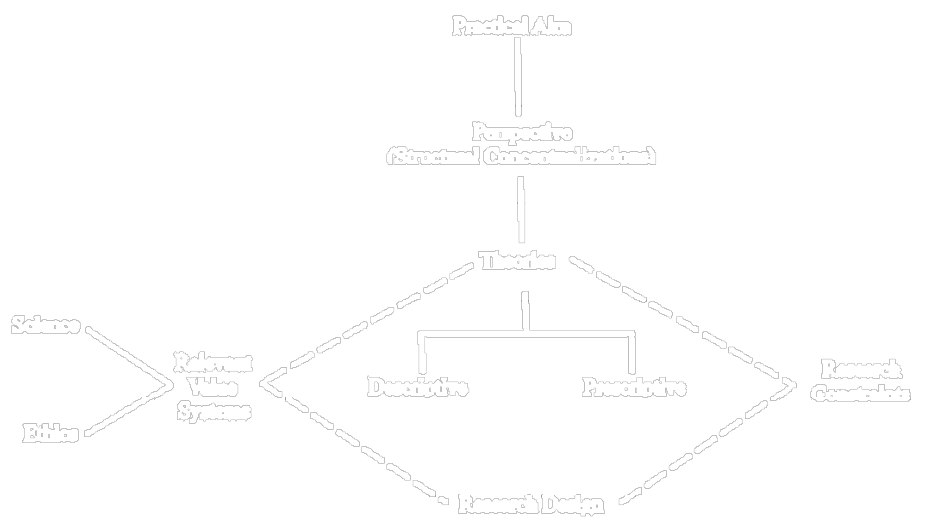

Figure 1:Tirée de la structure de la discipline professionnelle de Donaldson et Crowley (1) 
De plus, il devient possible de cerner la science infirmière et les théories issues d'un processus scientifique. La science infirmière a été influencée par les visions générales de la science, mais puisque les conceptions de la science sont nombreuses et qu'elles ont aussi évolué de façon importante au cours du XXe siècle, nous allons mettre de l'avant seulement deux visions qui ont exercé une influence importante sur l'évolution de la science infirmière. À partir de ces prémisses, nous proposons que l'avancement du savoir scientifique témoigne de l'avancement global de la discipline.

\section{Histoire de l'évolution de la dimension scientifique de la discipline infirmière}

Les progrès accomplis dans le développement scientifique de la discipline infirmière peuvent être relevés par une recension de la littérature. Cette évolution peut être découpée en différentes étapes. Par exemple, Meleis[5] suggère que le développement de la discipline a franchi les sept étapes suivantes: l'étape de la pratique, de l'éducation, de l'administration, de la recherche, de la théorie, celle de la philosophie et finalement, celle de l'intégration. On remarque que le développement de théories constitue en soi une étape du développement disciplinaire. Aussi, Debout[6] suggère trois périodes marquées par les éléments suivants : "la quête de théories, - la quête d'un paradigme, - la quête d'utilité clinique ".[6 p75] Ces auteurs, ainsi que la tendance observée dès 1972 chez la "National League for Nursing " à imposer dans ses normes d'accréditation que " tout programme de formation en sciences infirmières repose sur les théories de soins nouvellement produites ",[6 p75] montrent l'importance historiquement attribuée au développement de théories.

Aussi selon certains théoriciens comme Peplau[7], Henderson[8], Johnson[9], Abdellah[10], Donaldson et Crowley [1] et Schlotfeldt[4], la théorie est une composante essentielle dans la structure d'une discipline scientifique et académique. Pour toutes ces raisons, nous regroupons l'évolution de la discipline autour des théories et proposons une division en trois périodes : l'absence de théorie, la naissance de la théorie et la période de réflexion sur les théories.

\section{Absence de la théorie}

Un survol de l'histoire des soins infirmiers montre une première période où l'on note l'absence de théories et de travaux scientifiques. Néanmoins, on retrouve dans cette période les premiers écrits propres à la discipline, soit ceux de Florence Nightingale[11] à qui on attribue souvent l'amorce du développement de la discipline infirmière. En tant que membre de la classe supérieure dans son pays, Nightingale (1820-1910) a eu accès à une éducation et à une formation, ce qui lui aura sans doute permis de développer une conceptualisation des soins dans laquelle elle mettait l'accent sur le rôle de l'environnement dans la restauration de la santé. La formation qu'elle a mise sur pied vers la fin du 19 e siècle a orienté les programmes de formation implantés dans une centaine d'hôpitaux au début du 20e siècle, entrainant la véritable émergence du travail de théorisation des phénomènes spécifiques des soins grâce au contenu des programmes de formation.

\section{Naissance de la théorie}

Après l'impulsion initiale de Nightingale[11] à la fin du 19e siècle, il a fallu attendre le début des années 1950 pour que la définition de la substance de la discipline se clarifie pour la communauté infirmière et pour que des théoriciennes proposent des conceptions des soins infirmiers[5]. Cette période représentait tout un défi et a constitué justement une période de remise en question de ce que l'on entendait jusqu'à présent par "soins infirmiers ". Ainsi, pour certaines infirmières, mais également pour d'autres professionnels de la santé, les soins étaient simplement une partie de la médecine ou encore, ils faisaient partie des sciences biologiques, naturelles ou physiques. Selon Alligood[12], c'est à ce moment que les infirmières ont envisagé des manières distinctes de penser le soin en recourant à la théorie. Par exemple, l'Association des infirmières américaines (The American Nurses Association, ANA) a recommandé que le développement de théories soit priorisé pour les années 1966-1970.[5]

C'est dans cette foulée que des groupes d'infirmières ont été formés pour discuter de la nature des soins, de la nature du travail des infirmières et des aspects distinctifs des soins.[5] Leurs travaux visaient à articuler une vision théorique des soins en essayant de trouver des réponses aux questions portant sur la nature du soin, sa mission, ses buts et les rôles des infirmières. Ces premiers efforts ont conduit les théoriciennes à affirmer que le savoir des soins ne pouvait pas se réduire à celui issu des autres disciplines. Puis, au cours des années 1950 à 1980, les efforts des théoriciennes ont abouti au développement de théories infirmières dans lesquelles des phénomènes et des problèmes infirmiers sont abordés. Sous la direction combinée d'un groupe décisif [Nursing Theory Think Tank, 1979] et de théoriciennes, notamment Peplau[7], Henderson[8], Rogers[13], Roy[14] et Orem[15], on vit se développer du savoir théorique propre au domaine infirmier. Plus spécifiquement, certaines d'entre elles, comme 
Peplau[7], Henderson[8], Johnson[9] et Abdellah[10] ont écrit sur la nature de la théorie infirmière, les façons de l'élaborer et de la structurer. Pour ces dernières, la théorie est une composante essentielle d'une discipline scientifique et académique.

Par ailleurs, au cours de leurs travaux, ces théoriciennes se sont inspirées de différentes visions des sciences proposées par des philosophes comme Kuhn[16], Toulmin[17] et Popper[18]. Pour cette raison, les théories développées dans la discipline infirmière au cours des années 1960-1970 sont fortement influencées par les courants de philosophie des sciences populaires. Ainsi, la définition des phénomènes dans les théories infirmières au fil de ces années a généralement adopté une approche réductionniste et a eu recours à des méthodes de recherches visant à préserver l'objectivité en vue de saisir le phénomène en soi. Ce type d'approche scientifique découpe les phénomènes en composantes afin d'arriver à mieux les saisir pour ensuite en refaire une synthèse. Cela était associé à une vision particulière de la science courante à cette époque.

Ces visions ancrées dans des approches méthodologiques réductionnistes des phénomènes n'étaient cependant pas en mesure de répondre à toutes les questions soulevées dans la discipline infirmière et à la complexité de certains phénomènes au cœur des soins. Puisque les conceptions de la science ont évolué massivement à cette même période, elles ont apporté de nouvelles façons d'approcher les phénomènes des soins. Cela ne s'est pas fait sans controverses comme en témoignent diverses interprétations et la grande quantité de publications consacrées aux méthodes de recherche.[19] C'est dans cette foulée que certains chercheurs infirmiers se sont dirigés vers des méthodes qualitatives, notamment vers la phénoménologie.[67] Selon celles qui ont fait ce changement, cette approche leur semblait mieux saisir l'expérience de l'être humain et les phénomènes des soins. On note ainsi que les théories infirmières de la fin des années 1970 et celles des années 1980 se sont focalisées sur l'individu et son expérience, s'éloignant ainsi des méthodes précédentes. L'élaboration des théories s'inscrit résolument dans une vision holistique comme celles de Rogers[13] et de Parse[20].

L'apparition de théories a contribué à donner de la substance à la science infirmière puisque toutes les conceptions de la science de l'époque considèrent les théories comme inhérentes au travail de la science.[21] Ces théories visent à mieux préciser les soins en plus de proposer des visions de plusieurs phénomènes uniques aux soins. Le développement de ces premières théories marque l'entrée des infirmiers dans le champ de l'activité scientifique grâce à l'émergence d'un savoir spécifique et souligne la contribution d'infirmières américaines à l'élaboration des théories. À la suite de ces premiers efforts théoriques, les questionnements ont à nouveau surgi, mais cette fois à propos des composantes d'une théorie, des types de théories et des façons d'analyser et de critiquer les théories.[22]

\section{Période de la réflexion}

La période de réflexion a mobilisé les théoriciennes et les métathéoriciennes autour des quatre sujets suivants: le type de théories, le centre d'intérêt disciplinaire, la structure de la science infirmière et son avancement.

\section{Type de théories}

Si au début des années 1950, on doutait de la nécessité des théories infirmières, les travaux entrepris à partir des années 1960 montrent que ce n'est plus le cas alors que les théoriciennes se préoccupent désormais de préciser les caractéristiques d'une théorie,[23,24,25] ses composantes et la nature de son contenu principal[22]. Différentes façons d'analyser et de critiquer les théories sont proposées. [26] Au cours de cette période, Ellis[23], Dickoff, James et Wiendenbach[27] et Dickoff et James[28] se sont concentrés sur les façons de distinguer notamment les théories propres et celles empruntées. Cette préoccupation relevait du fait que parmi les théories utilisées, beaucoup étaient empruntées à d'autres disciplines et que l'on cherchait à comprendre ce qui caractérise une théorie infirmière comme celles développées par Peplau[7], Newman[29], Orlando[30] ou Watson[31]. De même, les discussions montrent différentes positions à propos des avantages et des inconvénients des théories empruntées ou partagées "Théories partagées a été conceptualisée selon une perspective infirmière si elle s'intègre de manière cohérente avec le phénomène d'intérêt central de la vision paradigmatique de la discipline infirmière et si elle s'avère empiriquement appropriée dans des situations de soins infirmiers" [38,39 p16]. Par exemple, Moore[32] affirme que l'utilisation des théories d'autres disciplines démontre la faiblesse de la discipline infirmière. Levine[33], Donaldson 34] et Chinn et Kramer[35] croient que cela constitue un riche réservoir où les infirmières puisent dans les savoirs disponibles pour comprendre la condition humaine. Parmi ces différentes positions, le point de vue de Fawcett[36] est différent et s'appuie sur une vision particulière de la théorie. Elle suggère que les théories empruntées peuvent contribuer à l'élaboration de la discipline infirmière si elles sont testées pour déterminer leur adéquation empirique dans 
des situations infirmières. Autrement dit, il faut choisir parmi les différentes théories, celles compatibles avec les buts visés par les soins infirmiers et ainsi, elles deviennent compatibles. Dans ce contexte, et devant la place occupée par les théories empruntées aux autres disciplines, on se questionnait au sujet de l'influence potentiellement négative des autres disciplines et des théories empruntées (la théorie empruntée signifie que le savoir a été développé par d'autres disciplines et a été extrait et utilisé par les infirmiers pour le soin [40]) sur le développement de la science infirmière, montrant que les défis de l'élaboration d'une vision distincte des soins infirmiers n'était pas disparue. C'est dans ce contexte que Fawcett[36] ajoute des critères permettant de déterminer si une théorie convient au domaine disciplinaire en se référant au but et au centre d'intérêt. Elle en fait des éléments primordiaux pour faire un choix de théorie en mesure de faire avancer la discipline infirmière.

\section{Éléments du centre d'intérêt}

Des années 1970-1980, les théoriciennes souhaitaient donner une direction aux théories qui jouent un rôle important dans l'avancement de la partie scientifique de la discipline. Suivant ce principe, Meleis[5] invitait les infirmières à recentrer le débat sur la substance de la discipline au lieu de sa syntaxe, soit sur le savoir à propos des phénomènes et les propositions théoriques centrales à la discipline. Les théoriciennes valorisent l'importance de définir la discipline et de cerner un domaine d'investigation marqué par cette perspective unique. À cet égard, une théorie convient au domaine disciplinaire si elle se réfère au centre d'intérêt.
Les propositions au sujet du centre d'intérêt et suggérées par différents auteurs ont été exposées dans différents livres comme ceux de Fawcett [38] et de Meleis [5, 40, 41]. Fawcett [38] a discuté de la vision d'une dizaine de théoriciennes et métathéoriciennes comme Newman [44], King [45] Parse [46], Donaldson et Crowley [1], Newman, Sime et CorcoranPerry [47], Meleis [41], Thorne et coll. [48], Newman [49], Barrett [50].

Dans la vision de Fawcett,[42] le métaparadigme incorpore les propositions de Donaldson et Crowley[1]. Plus spécifiquement, le métaparadigme comprend quatre concepts centraux [l'être humain, l'environnement, la santé et le soin] et quatre propositions qui les relient et qui constituent l'objet de la discipline.[51] Par ailleurs d'autres métathéoriciennes ont présenté des propositions différentes au sujet du centre d'intérêt de la discipline. Certaines comme Thorne et coll.[48], Newman[49], Barrett[50] ont proposé de reformuler le métaparadigme. Par exemple, Newman et coll.[47] proposent que "l'infirmière se centre davantage sur l'expérience de santé ou maladie comme telles ". Thorne et coll.[48] ajoutent que " les concepts centraux doivent refléter une neutralité paradigmatique et permettre une diversité de positions philosophiques ". Aussi, Newman [49] met l'accent sur le caractère entier de la personne [wholeness]. Au final et en général, on convient que la discipline s'intéresse au soin, à des personnes, à des familles, à des communautés et à des populations qui, en interaction continue avec leur environnement, vivent des expériences de santé. Pour certains, il s'agit moins d'un métaparadigme que d'une structure de base à partir de laquelle le savoir infirmier évolue.[52]

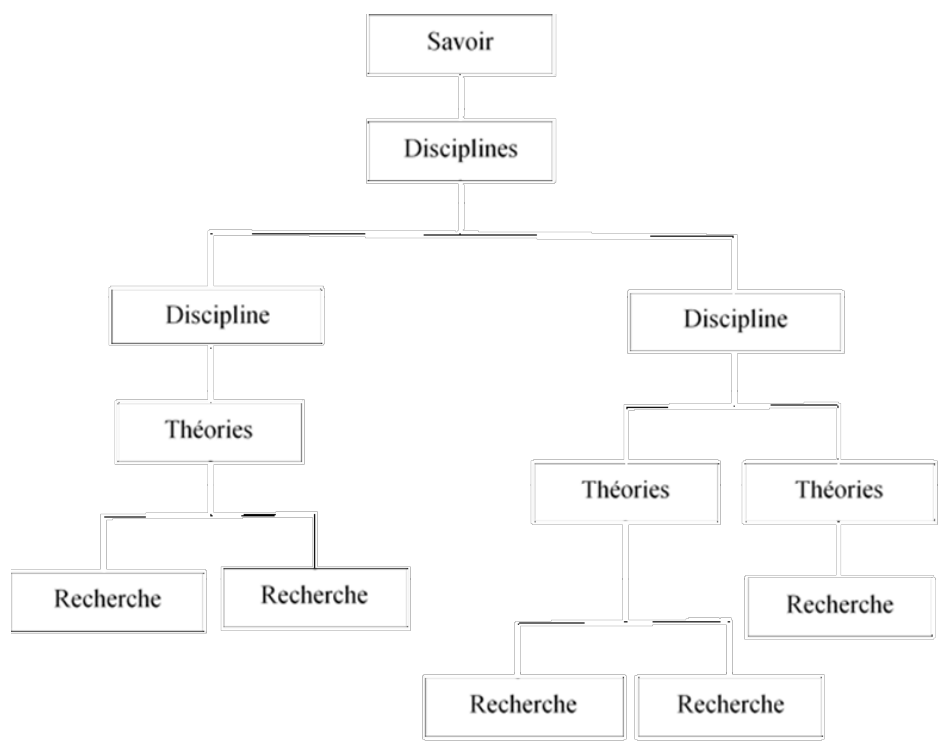

Figure 2: Tirée de la structure du savoir de Donaldson et Crowley (1978) (1) 
En somme, selon Fawcett[53], la formulation d'un métaparadigme pour la discipline infirmière représente un effort admirable pour consolider la discipline et recèle un énorme potentiel pour l'avancement du savoir infirmier et de la recherche[54]. La proposition d'un métaparadigme a été un élément important pour l'avancement théorique de la science infirmière en fournissant des repères pour cibler les phénomènes d'intérêt pour le développement de cette dernière.

\section{Faire avancer le savoir de la science infirmière}

Après avoir élaboré les premières conceptualisations des soins infirmiers et ce que les soins doivent être, I'histoire de la discipline montre que d'autres questions ont émergé chez les théoriciennes et les métathéoriciennes notamment : comment la dimension scientifique de la discipline devraitelle avancer? Outre leur proposition de structure du savoir, les métathéoriciennes comme Donaldson et Crowley[1] et Schlotfeldt[4] ont aussi proposé des façons de voir l'avancement du savoir. Donaldson et Crowley[1] ancrent leur vision dans le savoir de l'humanité et, pour cela, ils réunissent différents types de théories et de recherches en les situant dans une forme hiérarchique représentant les composantes d'une discipline professionnelle (Figure 2). Dans ce contexte, l'avancement du savoir d'une discipline, grâce à différentes approches systématiques de recherche, va produire des théories pour la discipline. Dans leur conception, on retrouve " des théories descriptives, issues de recherches fondamentales et appliquées et des théories prescriptives issues de recherches cliniques. Par conséquent, la discipline infirmière se fait grâce à la recherche fondamentale, la recherche appliquée et la recherche clinique ».[55, p13]
Par ailleurs, selon Schlotfeldt[4] la discipline, représentée par une grande sphère ayant une membrane perméable et extensible [représentée par la deuxième sphère], avance en complétant le noyau grâce à l'apport de nouvelles connaissances découvertes par des recherches systématiques et en effaçant les erreurs et les faussetés (Figure 3).

Bien que la structure de la discipline de Donaldson et Crowley[1] et celle de Schlotfeldt[4] soient différentes dans leur forme et dans certains de leurs éléments, il n'en demeure pas moins qu'elles sont comparables sur le plan de l'apport théorique issu d'une démarche scientifique pour son avancement. Dans les deux structures, l'avancement du savoir se fait selon le cheminement de la science qui est une partie importante de la discipline.

Comme nous l'avons vu dans la partie sur la structure de la discipline proposée par Donaldson et Crowley[1] et Schlotfeldt[4], l'avancement de la discipline se voit dans l'avancement du savoir scientifique infirmier. Plus généralement, ces auteurs suggèrent que si le savoir scientifique d'un domaine avance, une discipline survivra et avancera d'où l'importance de la question soulevée dans le présent article et la nécessité d'une vérification de l'influence exercée par des propositions spécifiques visant à assurer le développement de la discipline infirmière. Par ailleurs, tandis que les débats entre les métathéoriciennes sur les types des théories, le centre d'intérêt, la structure de la discipline et son avancement continuaient, d'autres points de vue ont été proposés pour répondre aux questions soulevées.

\section{Variations sur un même thème ou autres pistes pour le développement de la dimension scientifique de la discipline}

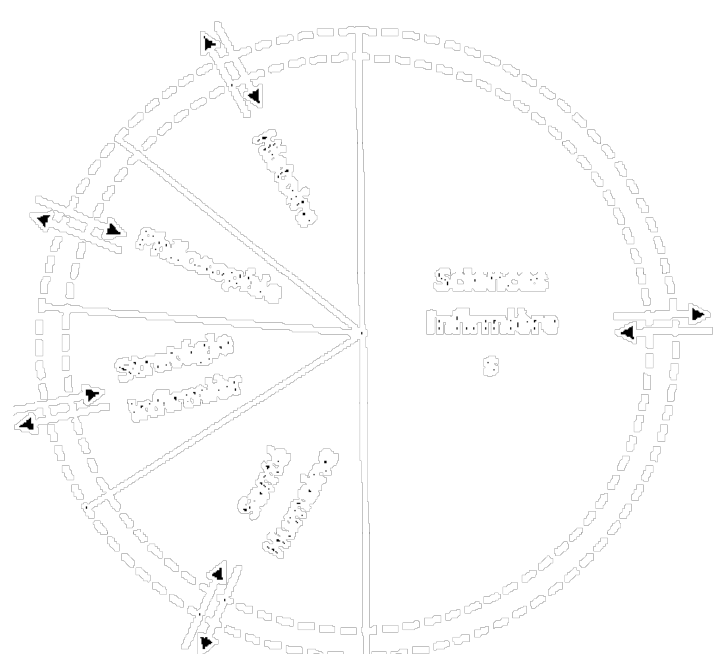

Figure 3 : Tirée de Schlotfeldt (1988) (4), la structure de la discipline infirmière 
En consultant la littérature infirmière et en élargissant la perspective sur la discipline dans sa globalité, on se rend compte qu'il existe plusieurs autres propositions que celles de Donaldson et Crowley[1] et de Schlotfeldt[4]. Selon Craig[56], les approches de développement du savoir infirmier peuvent être représentées sur un continuum s'étendant de l'approche scientifique pure de Martha Rogers[57] à l'approche pratique de Dickoff et James[28]. Ainsi, Roy et Jones[58] sont d'avis que la discipline avance, par la mise au point de conceptualisations, de perspectives philosophiques, de recherches méthodologiques et d'engagements sociopolitiques. Roy et Jones[58] ajoutent que les manifestations de progrès dans différentes zones de développement de la discipline infirmière, à l'image du développement de n'importe quelle autre discipline, sont interactives et itératives. Ainsi, les avancées dans une zone stimulent le mouvement dans une autre zone. À chaque fois, la spirale soutient un développement vers des niveaux théoriques plus élevés pour la discipline. Autrement dit, l'avancement du savoir scientifique contribue au développement général de la discipline. Meleis[5] présente les trois visions suivantes pour décrire l'avancement du savoir d'une discipline: révolution, évolution et intégration. La première est la théorie de révolution inspirée de la vision de Kuhn[59], selon laquelle les sciences se développent seulement pendant les périodes de crise. Pendant ces crises, les théories rivalisent, les anomalies sont identifiées et les inadéquations sont mises en évidence. La deuxième vision est une vision évolutive, où la science progresse dans la continuité et grâce à des changements cumulatifs à long terme. Finalement, la troisième vision est celle de l'intégration : selon celle-ci, le progrès de la discipline infirmière semble suivre son propre chemin; des idées rejetées dans une étape antérieure de développement sont acceptées à une étape différente.[5]

Gortner[60] a décrit le progrès de la discipline en retraçant l'identification et les travaux sur des phénomènes d'intérêt découlant de propositions issues de la recherche. Elle ajoute que les progrès dans l'élaboration du savoir ont influencé la maturation de la discipline infirmière. Elle souligne l'apport de différents éléments, notamment le passage de recherche isolée à des programmes de recherche, l'augmentation du nombre de programmes de doctorat qui préparent à la recherche et la diminution de discussions au sujet des méthodes de recherche.

Ces auteurs sont intéressants, mais l'auteure majeure demeure Jacqueline Fawcett. Fawcett[2] au cours des années 1970 et au début des années 1980, développait une approche pour répondre aux questions soulevées au sujet de la science infirmière en proposant une structure de la science infirmière intégrant son centre d'intérêt, sa structure, ses frontières, son contenu et proposait des voies pour l'avancement du savoir. Son point de vue a été largement diffusé et le fait qu'elle l'ait résumé d'une façon plus complète que les autres métathéoriciennes des années 1950 à 1980 pourrait expliquer sa renommée.

\section{Approche de Fawcett}

Jacqueline Fawcett[61], reconnue internationalement pour son travail métathéorique, a obtenu son $\mathrm{Ph}$. D. en sciences infirmières à I'Université de New York. Elle a consacré la majeure partie de sa carrière à l'élaboration et à l'implantation de son approche. Au cours des années 1970 et au début des années 1980, elle en est arrivée à retenir les quatre paramètres suivants comme indicateurs de l'avancement de la discipline : la spécification d'un métaparadigme en tant que centre d'intérêt, la définition de la structure du savoir infirmier pour lier les différentes dimensions de la discipline, une proposition pour son avancement et l'élaboration de modèles conceptuels infirmiers et de théories infirmières [2].

Après avoir lancé cette idée, Fawcett a précisé son approche en s'y concentrant pendant les 30 années suivantes. Fawcett[63] a tout d'abord retenu le terme de métaparadigme en s'inspirant de Kuhn[59] et de Dubin[64]. En bout de ligne, son approche préconise que le métaparadigme comprenne des concepts centraux [personne, santé, soin et environnement] et deux types de propositions : non relationnelles et relationnelles. Tandis que les propositions non relationnelles sont les définitions de chacun des quatre concepts du métaparadigme, les propositions relationnelles, dérivées du travail de Donaldson et Crowley[1] et de Gortner[60-65] expliquent les liens existants entre les concepts du métaparadigme[38-51]. Ensuite, elle a proposé le métaparadigme comme le premier élément d'une structure hiérarchique auquel elle ajoute les philosophies, les modèles conceptuels, les théories à spectre modéré et les indicateurs empiriques. Elle a aussi suggéré, dès 1984, une façon de les situer les uns par rapport aux autres en mettant les composantes du savoir infirmier dans une structure hiérarchique, structure qu'elle a par la suite transformée en une structure holarchique. Enfin, dans ses publications, elle a essayé de démontrer comment l'application de cette structure [conceptuelle-théorique-empirique [C-T-E]], et l'analyse effectuée en se basant sur cette structure pouvaient servir à montrer l'utilisation et l'utilité du savoir proposé. Toujours selon Fawcett [66], les modèles conceptuels sont les bases sur lesquelles les revendications de statut disciplinaire reposent puisqu'ils proposent des visions des soins infirmiers. Elle propose l'utilisation d'un modèle conceptuel afin de 
favoriser une meilleure cohérence. En effet, selon elle, le recours à un modèle conceptuel facilite la communication entre les infirmières et peut réduire les conflits entre elles en plus de fournir une approche systématique pour la recherche, l'éducation et l'administration [38]. Elle croit que l'avancement de la science infirmière, synonyme de discipline infirmière pour cette dernière, se réalisera en suivant ses propositions tout en conservant ses frontières avec d'autres disciplines.

L'explication de Fawcett[2,38] apparait complète, organisée et généralement acceptée. Autrement dit, le travail de Fawcett[2,38] ferait autorité et répondrait aux questions au sujet de l'avancement de la science infirmière. Ce n'est qu'en 2010 qu'une première remise en question formelle et structurée de la vision de Fawcett[2,38] est publiée. Risjord[3] présente une critique des propositions de Fawcett[51] et offre une vision différente du processus d'avancement de la dimension scientifique de la discipline infirmière.

Risjord[3] propose de voir le savoir organisé dans une structure en réseau dont les frontières sont plutôt floues facilitant son développement au pourtour du réseau. II présente aussi une vision qui considère les quatre mêmes éléments que Fawcett[51] : le centre d'intérêt, la structure du savoir infirmier pour lier différentes dimensions de la discipline et son évolution et les types des théories. On voit ainsi ressortir une différence davantage au niveau de la façon de faire avancer le savoir de la discipline, ce qui est intéressant.

\section{Approche de Risjord}

Mark Risjord[67] a reçu son baccalauréat en philosophie et en anthropologie de l'Université du Wisconsin et son doctorat en philosophie de l'Université de la Caroline du Nord. Après ses études en philosophie, il a proposé une approche pour le développement du savoir infirmier scientifique dans son volume qui s'intitule Nursing Knowledge Science, Practice, and Philosophy.

Dans sa vision, le centre d'unification de la discipline infirmière se réalise à partir du point de vue des soins (nursing standpoint). Selon Risjord[3], l'idée principale de ce point de vue (nursing standpoint) signifie que les recherches débutent à partir des questions des infirmières, puisque les savoirs infirmiers servent à résoudre des problèmes de la pratique et qu'essentiellement, les savoirs infirmiers sont des théories pour mieux comprendre les problèmes de la pratique. Pour lui, les théories s'organisent dans une structure en forme de réseau où les réponses aux problèmes soulevés à partir de la pratique arrivent à former des théories. De plus, les théories à spectre modéré créées par l'intersection des théories existantes sont en mesure de répondre aux questions posées par d'autres théories et constituent les théories les plus utiles pour la discipline. II croit que l'avancement de la science infirmière se réalisera tout en conservant ses frontières. II propose également que l'élaboration de théories à interniveaux (interlevel) serait le moyen le plus prometteur pour y arriver. Selon lui, ces théories peuvent contribuer au développement du savoir de la discipline [de façon horizontale] tout en situant le savoir infirmier par rapport au savoir des autres disciplines [de façon verticale]. De cette façon, les frontières de la discipline sont préservées, mais cette dernière se développe en restant connectée au savoir des autres disciplines et s'insère dans le réseau du savoir de l'humanité.

En somme, de Donaldson et Crowley[1], en passant par Schlotfeldt[4] et par les deux métathéoriciens, Fawcett[66] et Risjord[3], on voit qu'il existe plusieurs propositions en mesure de répondre aux questions soulevées au sujet du savoir infirmier scientifique, de sa structure et de son avancement. Au-delà de leurs différences, tous semblent s'entendre pour suggérer qu'une structure du savoir peut permettre de cibler le savoir scientifique et son évolution[3,66]. Or, l'étude de l'évolution de la science infirmière montre que deux types de structure du savoir, une structure hiérarchique et une concentrique, ont eu une influence durable sur l'évolution du savoir.

\section{Conclusion}

En somme, la discipline infirmière, branche du savoir humain, comprend des théories et des recherches sur des phénomènes qui représentent ses intérêts centraux. Selon la structure de la discipline présentée par Donaldson et Crowley[1] et Schlotfeldt[4], la théorie est une composante essentielle d'une discipline scientifique et académique. On peut ainsi examiner l'évolution de la discipline en trois périodes: l'absence de théorie, la naissance de la théorie et la période de réflexion sur les théories. La période d'absence de théorie comprend l'impulsion engendrée par les travaux théoriques de Nightingale[11]. Par la suite, l'émergence des théories se voit dans les différents travaux de théoriciennes[11] avant que s'amorce une période de réflexion et de remise en question à la suite des problèmes soulevés au fil des ans par l'apparition des théories et l'élaboration de la discipline. Plus récemment, les travaux de deux métathéoriciens, Fawcett[66] et Risjord 3] ont repris deux structures du savoir en mesure de proposer des réponses aux questions soulevées au sujet du savoir infirmier scientifique, de sa structure et de son avancement. Le fil conducteur de la structure du savoir et des théories permet de suivre l'évolution de la discipline infirmière.

L'examen de l'évolution de la science infirmière montre 
qu'elle est face à des propositions pour son avancement qui contiennent des éléments comparables. Une analyse plus approfondie de ces propositions pourrait stimuler le développement de la dimension scientifique de la discipline. Une telle piste est également intéressante d'un point de vue plus général, puisqu'on constate une lacune sur le plan d'analyses systématiques des propositions que l'on retrouve dans le domaine disciplinaire et, au final, cela pourrait dégager certaines orientations.

\section{Références}

1.Donaldson, S.K. et Crowley, D.M. The discipline of nursing. Nursing Outlook, 1978, 26 [2], 113-120.

2.Fawcett, J. Hallmarks of success in nursing theory development. In P.L. Chinn [Ed], Advances in Nursing Theory development, Rockville, MD: Aspen, 1983, pp.3-17.

3.Risjord, M. Nursing Knowledge, Science, Practice, and Philosophy, 2010, 1 ed., Wiley-Blackwell.

4.Schlotfeldt, R. Structuring nursing knowledge: A priority for creating nursing's future. Nursing Science Quarterly, 1989, $1[1]: 35-38$.

5.Meleis, A.I. Theoretical Nursing. Theoretical nursing, 2012, Development \& Progress. 5th ed. Philadelphia: Lippincott Williams \& Wilkins.

6.Debout, Ch. Sciences des soins infirmiers: réflexions épistémologiques sur le projet d'une discipline. [2008], Dans Recherche en soins infirmiers. 2 [N 93], pages 72 à 82.

7.Peplau, H.E. Interpersonal relations in nursing, 1952, New York: G.P. Putnam's Sons.

8. Henderson, V. Nature of nursing, 1966, New York: Macmillan.

9.Johnson, D.E. A philosophy of nursing. Nursing Outlook, 1959, 7[4], 198- 200.

10.Abdellah, F.G. The nature of nursing science. Nursing Research, 1969, 18, 390-393.

11] Nightingale, F. Notes on nursing: What it is and what it is not. 1946, Philadelphia: J.B. Lippincott.

12] Raile Alligood, M. Introduction to Nursing Theory: Its History, Significance, and Analysis. 2014, In Evolution of Nursing Theories. Consulté à l'adresse : http://www. elsevieradvantage.com/samplechapters/9780323091947/ AlligoodCh1-9780323091947.pdf.

13.Rogers, M. An introduction to the theoretical basis of nursing, 1970, Philadelphia: F.A. Davis.
14.Roy, C. Adaptation: A conceptual framework for nursing Nursing Outlook, 1970, 18, 42-48.

15.Orem, D.E. Nursing: Concepts of practice, 1971, New York: McGraw-Hill.

16.Kuhn, T. S. The Structure of Scientific Revolutions [2nd ed.]. 1970, Chicago: University of Chicago Press.

17.Toulmin, S. Human Understanding: The Collective Use and Evolution of Concepts. 1977. Princeton, NJ: Princeton University Press.

18.Popper, K.R. La connaissance objective, [1978], traduit de l'anglais par Catherine Bastyns, Presses Universitaires de France. pp.7, 31-35, 69, 135.

19.Cody, W.K. Paradigm shift or paradigm drift? A meditation on commitment and transcendence. Nursing Science Quarterly, 2000, 13[2], 93-102.

20.Parse, R.R. Nursing science: Major paradigms, theory and critiques. 1987, Philadelphia: W. B. Saunders.

21.Nadeau, M.A. L'évaluation de programme théorie et pratique, 1988, 2 édition, Les presses de l'université Laval.

22.Jacox, A. Theory construction in nursing: an overview. Nursing Research, 1974, 23[1], 4-13.

23.Ellis, R. Characteristics of significant theories. Nursing Research, 1968, 17[3], 217-222.

24.Ellis, R. Reaction to Walker's article. Nursing Research, 1971, 20 [6], 494.

25.Walker, L.O. Toward a clearer understanding of the concept of nursing theory. Nursing Research, 1971, 20 [5], 428-435.

26.Duffey, M.et Muhlenkamp, A.F. A framework for theory analysis. Nursing Outlook 1974, 22[9], 570-574.

27.Dickoff, J., James P. et Wiendenbach, E. Theory in a practice discipline. Nursing Research, 1968a, 17:415-435.

28.Dickoff, J. et James, P. A theory of theories: A position paper. Nursing Research, 1968b, 17, 197-203.

29.Newman, MA. Health as Expanding Consciousness. 1986, St. Louis, MO: C V Mosby.

30.Orlando, I.J. The dynamic nurse-patient relation: function, process and principle. [1961], New York: G.P. Putnam's Sons.

31.Watson, J. Nursing: The philosophy and science of caring [pp.9-10]. 1979, Boston: Little, Brown.

32.Moore, S. Thoughts on the discipline of nursing as we approach the year 2000. Journal of Advanced Nursing, 1990, 
$15,825-828$.

33.Levine, M.E. The rhetoric of nursing theory. Image: Journal of Nursing Scholarship, 1995, 27, 11-14.

34.Donaldson, S.K. Breakthroughs in scientific research. The discipline of nursing: 1960-1999. Annual Review of Nursing Research, 2000, 18: 247-311.

35.Chinn, P. et Kramer, M. Theory and nursing: a systematic approach, 1995, 5th ed, Mosby Year Book, St Louis.

36.Fawcett, J. The relationship of theory and research, 1999, 3 ed. Philadelphia: F.A. Davis. pp. $28-58$.

37.Jovic, L., Lecordier, D., Poisson, M., Vigil-Ripoche, M.A., Delon, B. , Mottaz, A.M., Bourkia, D. , Joutard, Thierry et Tenza, M. L'enseignement des sciences infirmières en France : contenus et stratégies. Dans Recherche en soins infirmiers 2014/4 [N¹19], pages 8 à 40.

38.Fawcett, J. Contemporary Nursing Knowledge. Analysis and Evaluation of Nursing Models and Theories. 2005, Philadelphia: F.A. Davis.

39. Desbiens, J.F. Développement et validation d'une mesure de perception de compétence infirmière en soin palliatifs. 2011, Thèse présentée à la Faculté des études supérieures de I'Université Laval dans le cadre du programme de doctorat en sciences infirmières pour l'obtention du grade de Philosophiae Doctor [Ph.D.]. Faculté des sciences infirmières, Université Laval. Québec.

40.Meleis, A.I. Theoretical nursing: development and progress, 2007, 4 ed. Wolters Kluwer, Lippincott Williams \& Wilkins.

41.Meleis, A.I. Theoretical nursing: Development and progress, 1997, [3rd]. Philadelphia: Lippincott.

42.Fawcett, J. The relationship between theory and research: double helix. ANS: Advances in Nursing Science, 1978, 1[1], 36-39.

43. Fawcett, J. The Roy Adaptation Model and content analysis. Aquichan, 2006, 6[1], 34-37.

44.Newman, M.A. 'Newman's health theory' dans I. Clements, F. Roberts [Eds] to Nursing Care, New York, Family Health: A Theoretical Approach, 1983, York, John Wiley \& Sons, p. 161175.

45.King, I.M. Philosophy of nursing education: A national survey, Western Journal of Nursing Research, 1984, 6, 387406.

46.Parse, R.R. Transforming research and practice with the human becoming theory. Nursing Science Quarterly, 1997,
$10[4], 171-174$.

47.Newman, M.A., Sime, A.M. and Corcoran-Perry, S.A. The Focus of the Discipline of Nursing, Advances in Nursing Science, 1991, 14/1, p. 1-6.

48.Thorne, S., Canam, C., Dahinten, S., Hall, W., Henderson, A., et Kirkham, S. Nursing's metaparadigm concepts: Disimpacting the debates. Journal of Advanced Nursing, 1998, 27, 1257-1268.

49.Newman, M.A. The pattern that connects. Advances in Nursing Science, 2002, 24[3], 1-7.

50.Barrett, E.A.M. What is nursing science? Nursing Science Quarterly, 2002, 15, 51- 60.

51.Fawcett, J. The metaparadigm of nursing: Current status and future refinements. Image: Journal of nursing scholarship, 1984, 16, 84-87.

52.Chick, N., et Meleis, A.I. Transitions: A nursing concern. In P.L. Chinn [Ed.]. À partir de : Recherche en Soins Infirmiers, 1986, $n^{\circ}$ 63, décembre 2000 et Jacinthe Pépin et al., La Pensée infirmière, op. cit.

53.Fawcett, J. The metaparadigm of nursing: Current status and future refinements. Image: Journal of nursing scholarship, 1984, 16, 84-87.

54.Brody, J. A response to Dr. J. Fawcett's paper: "The Metaparadigm of Nursing: Present Status and Future Refinements", Image, The Journal of Nursing, 1984, 16[3], 87-89.

55.Dallaire, C.et Aubin, K. Les soins infirmiers, les sciences infirmières ou la science infirmière. Ch. 1. Dans Dallaire, C. [ed.], Le savoir infirmier: au cœur de la discipline et de la profession infirmière. Gaëtan.

56.Craig, S.L. Theory development and its relevance for nursing, Journal of Advanced Nursing, [1980], Vol. 5 [4], 349 -355 .

57.Rogers, M.E. Nursing: to be or not to be? Nursing Outlook, 1972, 20, 42-46.Morin: 2008, Boucherville. Pp.3-26.

58.Roy S.C. et Jones, D.A. Nursing Knowledge Development and Clinical Practice, 2007, springer publishing company, New York.

59.Kuhn, T.S. The essential tension: selected studies in scientific tradition and change, 1977, Chicago: University of Chicago press.

60.Gortner, S. R. Knowledge development in nursing: our historical roots and future opportunities. Nursing Outlook, 
2000, 48[2], 60-67.

61.Fawcett: https://www.ulaval.ca/notre-universite/prixet-distinctions/doctorats-honoris-causa-de-luniversitelaval/recipiendaires-doctorat-honoris-causa-2012-2013/ jacqueline-fawcett.html.

62.Lee, J. et Fawcett, J. The Influence of the Metaparadigm of Nursing on Professional Identity, Nursing Science Quarterly, 2013, 26[1], 96-98.

63.Fawcett, J. The relationship between identification and patterns of change in spouses "body image during and after pregnancy, International Journal of Nursing Studies, 1977, 14, 199-213.

64.Dubin, R. Theory building, 1969, New York: Free Press.

65.Gortner, S. Nursing science in transition. Nurs Research; 1980, 29:180 -3.

66. Fawcett, J. Conceptual Models of nursing: International in scope and Substance? The Case of the Roy Adaptation Model, Nursing Science Quarterly, 2003, 16:4, pp. 315- 318.

67.Risjord, M. http://ila.emory.edu/_includes/documents/ sections/people/Risjord\%20Vita.pdf.

68.Sylvain, H. Le devis constructiviste : une méthodologie de choix en sciences infirmières, L'infirmière clinicienne, 2008, Vol.5, no1, pp.1-11.

Pour contacter les auteures:

Farzaneh Farshbaf Kamel inf. PhD

Université Laval

Faculté des sciences infirmières,

Québec, Canada, G1V 0A6

couriel : farzaneh.farshbaf-kamel.1@ulaval.ca

Clémence Dallaire inf. PhD

Professeure titulaire

Université Laval

Faculté des sciences infirmières 